METZLER LEXIKON

ENGLISCHSPRACHIGER AUTORINNEN UND AUTOREN 


\title{
METZLER LEXIKON ENGLISCHSPRACHIGER AUTORINNEN UND AUTOREN
}

\author{
Herausgegeben von \\ Eberhard Kreutzer und Ansgar Nünning
}

631 Porträts

Von den Anfängen bis in die Gegenwart

Verlag J. B. Metzler

Stuttgart · Weimar 


\title{
Inhaltsverzeichnis
}

\author{
Vorwort $v$ \\ Autorinnen und Autoren A-Z $\quad 1-647$ \\ Literaturgeschichten und Nachschlagewerke 648 \\ Verzeichnis der Mitarbeiterinnen und Mitarbeiter 650 \\ Personenregister 655
}

\section{Die Herausgeber}

Eberhard Kreutzer; Professor für Englische Philologie an der Universität Bonn; Forschungsschwerpunkte: englische Literatur seit dem 18. Jh., amerikanische Literatur seit dem 19. Jh., irische und neue englischsprachige Literaturen des 20. Jh. Bei J.B. Metzler ist erschienen: Englische Literaturgeschichte. 3. Auflage 1999 (Mitautor).

Ansgar Nünning; Professor für Englische und Amerikanische Literatur- und Kulturwissenschaft an der Universität Gießen; Veröffentlichungen u.a zur englischen Literatur des 17. bis 20. Jh. sowie zu literaturund kulturtheoretischen Ansätzen. Bei J.B. Metzler ist erschienen: Metzler Lexikon Literatur- und Kulturtheorie. 2. Auflage 2001 (Herausgeber).

Die Deutsche Bibliothek - Cip-Einheitsaufnahme

Metzler-Lexikon englischsprachiger Autorinnen und Autoren : 631 Porträts ; von den Anfängen bis in die Gegenwart / hrsg. von Eberhard Kreutzer und Ansgar Nünning. - Stuttgart ; Weimar : Metzler, 2002

ISBN 978-3-476-01746-8

ISBN 978-3-476-01746-8

ISBN 978-3-476-02803-7 (eBook)

DOI 10.1007/978-3-476-02803-7

Dieses Werk einschließlich aller seiner Teile ist urheberrechtlich geschützt. Jede Verwertung außerhalb der engen Grenzen des Urheberrechtsgesetzes ist ohne Zustimmung des Verlages unzulässig und strafbar. Das gilt insbesondere für Vervielfältigungen, Übersetzungen, Mikroverfilmungen und die Einspeicherung und Verarbeitung in elektronischen Systemen.

(C) 2002 Springer-Verlag GmbH Deutschland

Ursprünglich erschienen bei J.B. Metzlersche Verlagsbuchhandlung und

Carl Ernst Poeschel Verlag GmbH in Stuttgart 2002 


\section{Vorwort}

Die englischsprachigen Literaturen erfreuen sich auch hierzulande seit langem besonders großer Beliebtheit. Dies liegt nicht allein an den Jahrhunderte zurückreichenden, intensiven deutschenglischen Literaturbeziehungen, die dazu beigetragen haben, daß eine Vielzahl bedeutender englischer Autorinnen und Autoren auch in Deutschland zu den Klassikern zählt, sondern auch an der intensiven Rezeption vieler Autor/inn/en der Moderne und Postmoderne. $\mathrm{Zu}$ den auch bei uns vielgelesenen Klassikern zählen daher sowohl Shakespeare, Milton und die Dichter der Romantik, die großen Erzähler vom 18. Jahrhundert bis zur Vormoderne wie Daniel Defoe, Henry Fielding, Samuel Richardson, Laurence Sterne, Jane Austen, Sir Walter Scott, die Brontë-Schwestern, Charles Dickens, William Makepeace Thackeray, George Eliot, Thomas Hardy und Joseph Conrad, Ikonen des 20. Jahrhunderts wie James Joyce, D. H. Lawrence, Virginia Woolf, T.S. Eliot und Samuel Beckett als auch Autor/inn/en der populären Genres wie Barbara Cartland, Agatha Christie, Ian Fleming, John Le Carré und die Verfasserin der Harry Potter-Romane, J.K. Rowling, um nur einige wenige zu nennen. Außerdem haben gerade auch die Welterfolge vieler zeitgenössischer englischsprachiger Autor/inn/en - z. B. Alan Ayckbourn, Julian Barnes, Antonia S. Byatt, John Fowles, William Golding, Nick Hornby, Kazuo Ishiguro, Doris Lessing, Ian McEwan, Harold Pinter, Peter Shaffer, Graham Swift, Barry Unsworth und Jeanette Winterson - dafür gesorgt, daß die "britische Literaturszene [...] derzeit als aufregendste in Europa" gilt, wie vor einigen Jahren Der Spiegel bemerkte: „Dieser Boom der britischen Literatur ist vor allem in ihrer Vielfalt begründet, im Nebeneinander von Stilrichtungen und Themen, von Experimentellem und nahezu Klassischem, dem Nebeneinander auch von Generationen und Kulturen." (23. Juni 1997, S. 160-162).

Die große internationale Popularität der englischsprachigen Literatur ist v.a. auch darauf zurückzuführen, daß Autor/inn/en aus den ehemaligen Kolonien inzwischen einen festen Platz in der britischen Literaturszene haben und daß die anglophonen Literaturen außerhalb Großbritanniens weltweite Beachtung gefunden haben. Es sind gerade diese postkolonialen $>$ Neuen Englischspra- chigen Literaturen (New English Literatures bzw. genauer New Literatures in English), denen die renglischer Literatur in den letzten Jahrzehnten viele wichtige Impulse und ihre große internationale Resonanz verdankt. Diese Sammelbezeichnungen beziehen sich auf die anglophonen Literaturen, die im Bereich des ehemaligen britischen Kolonialreichs von Kanada und der Karibik über Afrika und Südasien bis Australien und Neuseeland geschrieben werden und die vielfach auch in Großbritannien und Deutschland großen Anklang finden. Daß seit 1980 mit Salman Rushdie, Thomas Keneally, J.M. Coetzee, Keri Hulme, Peter Carey, Ben Okri, Michael Ondaatje, Roddy Doyle, Patrick McCabe, James Kelman, Arundhati Roy und Margaret Atwood über ein Dutzend nichtenglische Autor/inn/en mit dem renommierten Booker Prize ausgezeichnet wurden, macht deutlich, wie international die renglischer Literatur geworden ist. Stellvertretend für eine Vielzahl anderer Autor/inn/en seien außerdem Chinua Achebe, Janet Frame, Athol Fugard, Amitav Ghosh, David Malouf, Rohinton Mistry, Alice Munro, Ngugi wa Thiong'o, Caryl Phillips und Zadie Smith sowie die Nobelpreisträger/innen $\mathrm{Na}$ dine Gordimer, Wole Soyinka, Derek Walcott, Patrick White und zuletzt V.S. Naipaul genannt, die der englischsprachigen Literatur eine Bedeutung verliehen haben, die manche Kritiker vom Beginn einer neuen 'Weltliteratur/ sprechen lassen. Nicht zu vergessen ist in diesem Zusammenhang aber auch der mit Jonathan Swift einsetzende, seit dem ausgehenden 19. Jahrhundert sich verstärkende und insgesamt bemerkenswerte Beitrag irischer Autor/inn/en - von W. B. Yeats und George Bernard Shaw über Joyce und Beckett bis zu Seamus Heaney - zur englischsprachigen Literatur, die enorme literarische Produktivität der lange im repressiven Schatten Großbritanniens stehenden Nachbarinsel, die nicht von ungefähr anderen postkolonialen Literaturen Modelle geliefert hat.

Dieses Lexikon präsentiert in rund 630 Porträts englischsprachige Autorinnen und Autoren vom frühen Mittelalter bis zur unmittelbaren Gegenwart. Es versteht sich als wesentliche Ergänzung zu dem bereits erschienenen Metzler Lexikon Amerikanischer Autoren (Stuttgart: J.B. Metzler Verlag 2000), das ausschließlich Autor/inn/en der USA 
gewidmet ist. Die von ausgewiesenen Literaturwissenschaftlerinnen und -wissenschaftlern verfaßten Porträts konzentrieren sich auf das Leben und Werk der Autor/inn/en, berücksichtigen aber auch den literaturgeschichtlichen Kontext und auBerliterarische Aspekte. Ausgehend von den biographischen Daten informieren die Artikel über die bevorzugten Gattungen sowie über die Hauptwerke und deren Rezeption. Aufschlüsse über die Nachwirkung geben auch die nach den Originaltiteln vermerkten deutschen Übersetzungen. Bibliographische Hinweise auf größere Werkausgaben und weiterführende Sekundärliteratur schließen die Beiträge ab.

Die Autorenporträts zielen besonders auch darauf ab, einen Eindruck von der Individualität der jeweiligen Lebensumstände und Werke der einzelnen Autor/inn/en zu vermitteln. Innerhalb des skizzierten Rahmens haben wir es daher den Beiträgerinnen und Beiträgern überlassen, eigene $\mathrm{Ak}$ zente $\mathrm{zu}$ setzen und sich auf charakteristische, exemplarisch für das Ganze stehende Aspekte aus Leben und Werk zu konzentrieren, und bewußt darauf verzichtet, alle Artikel in ein einheitliches Leben-Werk-Wirkung-Schema zu pressen oder enzyklopädische Vollständigkeit von Lebens- und Werkdaten anzustreben. Über seine Funktion als biographische, literaturgeschichtliche und bibliographische Informationsquelle hinaus möchte dieses Lexikon auf diese Weise auch 'Lust` an der weiterführenden Lektüre vermitteln, Interesse an gerade auch zeitgenössischen - Autor/inn/en und ihren Werken wecken sowie zur vertiefenden Beschäftigung und zu bibliophilen Entdeckungsreisen anregen.

Ein weiteres Anliegen dieses Lexikons besteht darin, nicht nur die Klassiker der englischen Literatur vorzustellen, sondern zugleich einen möglichst breit angelegten und facettenreichen, wenngleich notgedrungen selektiven Úberblick über die Vielfalt der englischsprachigen Literaturen zu ermöglichen. Die Auswahl der Autorenporträts orientiert sich daher zum einen am traditionellen Kanon der englischen Literaturgeschichte und berücksichtigt alle 'Klassiker` von Chaucer und Shakespeare über Byron, Coleridge, Wordsworth, Keats, Shelley und Tennyson bis zu Joyce und Virginia Woolf, denen entsprechend längere Artikel gewidmet sind. Zum anderen ging es uns jedoch darum, diesen Kanon zu aktualisieren und in mehrfacher Hinsicht zu erweitern:

- erstens durch die Einbeziehung einer großen
Zahl von zu Unrecht vergessenen oder vernachlässigten Autorinnen, die vielfach erst in den letzten Jahrzehnten von der feministischen Literaturgeschichtsschreibung wiederentdeckt wurden und die oftmals selbst in neuen Literaturgeschichten, in denen sich meist nur eine verschwindend kleine Anzahl von Schriftstellerinnen findet, noch nicht angemessen repräsentiert sind;

- zweitens durch die Aufnahme möglichst vieler Autor/inn/en aus dem globalen Einzugsbereich der New English Literatures bzw. 'postkolonialen Literaturen von Kanada bis Neuseeland, zu dem in gewisser Hinsicht auch Irland zu zählen ist;

- und drittens durch die großzügige Berücksichtigung auch jüngerer zeitgenössischer Autor/inn/ en, die bislang noch kaum in vergleichbaren Nachschlagewerken zu finden sind.

Der skizzierten Zielsetzung entsprechend, legt das Lexikon einen relativ weitgefaßten Literaturbegriff zugrunde, der u.a. auch die Genres der Essayistik, Reiseliteratur, Philosophie, Autobiographie und Geschichtsschreibung sowie der Kinderbuchliteratur, des Kriminalromans, des Spionageromans und des 'Trivialromans` umfaßt. Neben den Klassikern des englischen Dramas, Romans und der Lyrik finden sich daher zum einen auch Schriftsteller/innen wie George Berkeley, Edward Gibbon, Thomas Hobbes, David Hume, John Maynard Keynes, John Locke, Thomas Babington Macaulay, John Stuart Mill, John Henry Newman, Adam Smith und Mary Wollstonecraft, zum anderen aber auch Autor/inn/en wie Enid Blyton, Lewis Carroll, Bruce Chatwin, G.K. Chesterton, Agatha Christie, Roald Dahl, Len Deighton, Arthur Conan Doyle, Ken Follett, Jerome K. Jerome, Edward Lear, Alan Alexander Milne, Ruth Rendell und J.R.R. Tolkien.

Es liegt auf der Hand, daß diese Auswahl ebenso wie jede andere - angreifbar ist. Bei der Auswahl der 631 aus einer zunächst über 2500 Namen umfassenden Liste von Autor/inn/en haben wir uns freilich weniger von persönlichen Vorlieben leiten lassen als von einigen Kriterien, die als Gradmesser der Kanonisierung und Rezeption von Autor/inn/en und ihrer Werke gelten können und die die getroffene Auswahl zumindest bis zu einem gewissen Grade nachvollziehbar machen sollen. Aufgenommen wurden v.a. solche Autor/inn/en, die zum einen in den meisten Literaturgeschichten und Nachschlagewerken sowie 
der heutigen Forschung figurieren und die zum anderen auch im deutschsprachigen Raum rezipiert worden sind, was sich u. a. an Übersetzungen und deutschen Werkausgaben ablesen läßt. Bei der selbst für Fachleute kaum noch überschaubaren Vielfalt der zeitgenössischen Literatur - gerade auch im Bereich der Neuen Englischsprachigen Literaturen - haben wir uns um eine repräsentative Auswahl von Autor/inn/en aus den verschiedenen Kulturkreisen bemüht und insbesondere solche aufgenommen, die auch hierzulande schon rezipiert worden sind (was beispielsweise in der zeitgenössischen Literatur weit mehr für Erzähler/innen als für Lyriker/innen gilt). Auch haben wir uns von dem pragmatischen Gedanken leiten lassen, daß ein Lexikon - im Unterschied zu Überblicksdarstellungen - nicht der Ort für die Vorstellung weithin unbekannter Autor/inn/en ist: Wer im deutschsprachigen Raum noch skeinen Namen hat‘, wird von den Benutzer/inne/n eines Lexikons auch nicht nachgeschlagen. Auf diese Weise waren wir bestrebt, uns maßgeblich an den Interessen und Informationsbedürfnissen des (nicht nur auf Fachleute beschränkten) Zielpublikums zu orientieren. Daß dennoch manch eine Leserin und manch ein Leser die eine Autorin oder den anderen Autor vermissen werden, ist freilich unvermeidlich. Am liebsten hätten auch wir die doppelte oder dreifache Anzahl von Porträts aufgenommen, was aber den begrenzten Umfang eines solchen Nachschlagewerkes gesprengt hätte. Daß eine Handvoll Autor/inn/en nicht in diesem Lexikon repräsentiert ist, entzieht sich zudem insofern unserer Verantwortung, als uns ein paar Beiträger/innen trotz zahlloser Zusicherungen (und Ermahnungen unsererseits) doch im letzten Moment im Stich gelassen haben und wir trotz der großen Kooperationsbereitschaft einiger kurzfristig eingesprungener Beiträger/innen nicht alle Lücken schließen konnten.
Der großen Mehrzahl der 220 Beiträgerinnen und Beiträger, die die Artikel für dieses Lexikon geschrieben haben, sind wir freilich für die ebenso ertragreiche wie zuverlässige Zusammenarbeit zu größtem Dank verpflichtet. Ohne deren Fachkompetenz, Belesenheit, Disziplin und geduldige Bereitschaft, umgehend auf Rückfragen, Vorschläge und Kürzungswünsche zu reagieren, wäre das termingerechte Erscheinen dieses Lexikons nicht möglich gewesen. Ebenso herzlich danken möchten wir den Herausgebern des PParallelbandes Metzler Lexikon Amerikanischer Autoren, unseren äußerst hilfsbereiten Kollegen Bernd Engler (Tübingen) und Kurt Müller (Jena), die uns insbesondere in der Anfangsphase mit Rat und Tat zur Seite gestanden haben, sowie Ute Hechtfischer, Bernd Lutz und Oliver Schütze vom Metzler Verlag, die uns in allen Phasen unterstützt haben. Ganz besonderer Dank gebührt schließlich Wibke Bindemann, Hanne Birk, Britta Freitag, Stefanie Hoth, Evelyn Kreutzer, Stephan Kreutzer, Rosemary Lawson, Julijana Nadj, Dagmar Sims und Katja Zinn sowie v. a. Gaby Allrath, Klaudia Seibel und Carola Surkamp, die für ihre Akribie, gewissenhafte Hilfe und unübertroffene Zuverlässigkeit und nicht zuletzt ihre Mühe bei der Erstellung eines druckreifen Manuskripts allergrößtes Lob verdient haben. Auch die termingerechte Fertigstellung dieses Bandes ist nicht nur der engagierten Mitarbeit der Beiträgerinnen und Beiträger zu verdanken, sondern auch dem unermüdlichen Einsatz der namentlich Genannten. Gewidmet sei der Band in Dankbarkeit dem Andenken des groBen Freiburger Anglisten Willi Erzgräber, der die letzten Artikel seines so produktiven und erfüllten Lebens für dieses Lexikon geschrieben hat.

Bonn und Gießen, im April 2002 Eberhard Kreutzer und Ansgar Nünning 